\title{
Scanning Electron Diffraction - Crystal Mapping at the Nanoscale
}

\author{
Paul Midgley ${ }^{1}$ and Duncan Johnstone ${ }^{1}$
}

1. Department of Materials Science and Metallurgy, University of Cambridge, Cambridge, UK.

Advances in microscope control, novel fast, efficient spectrometers and cameras and the rapid increase in computational power have combined to enable the development of new 'multi-dimensional' techniques and to open up many new avenues in their application across a wide spectrum of materials.

One key area that is rapidly evolving is at the interface between imaging and diffraction. For many years, scanning transmission electron microscopy has been used to record almost directly interpretable images using the high angle annular dark field signal. Typically, this has been accomplished using a single annular detector integrating the signal over a large scattering angle. More recently, with the introduction of pixelated detectors and fast data storage, electron diffraction (CBED) patterns have been recorded at every scanned pixel so as to build up a 4D STEM data set. If the STEM probe is coherent and with a sufficiently large convergence angle to produce disc overlap in the CBED pattern, techniques such as ptychography may be used to recover the phase and amplitude of the exit wave function and, in favourable cases, this can reveal the projected potential of the region of interest [1].

In the electron diffraction community, a similar technique has evolved known as scanning electron diffraction (SED) in which again a 4D data set is recorded with diffraction patterns acquired at every real space pixel. However, unlike in the case describe above, for most SED experiments the probe is near-parallel so as to give 'spot-like' patterns and with a probe size that is, to a large extent, diffraction limited. Moreover, the applications of SED, and the samples studied, differ from those explored with 4D-STEM in that they are typically thicker (where dynamical effects are likely to play a role), the regions of interest may be extended (perhaps over several microns) and where crystal imperfection (e.g. strain) is likely to be heterogeneous and three dimensional. For these samples, analysis of the geometry of the diffraction pattern provides a wealth of information that can be used to determine local orientations, strain, phase identification, etc [2]. Nevertheless, there is no doubt that the SED and 4D STEM communities are converging and the boundaries between the techniques becoming blurred.

In this paper, we will illustrate recent advances in the development of SED and its applications across a wide area of materials science. The speed with which individual diffraction patterns may be recorded (few ms) enables local crystallographic data to be acquired from even the most beam sensitive of material (e.g. organics, MOFs, pharmaceuticals, polymers) [3].

SED is also an effective technique to study low-dimensional materials, such as graphene. Figure 1(a) shows an orientation map determined using the open-source pyXem software at www.github.com/pyxem/pyXem. The rotation of the pattern from pixel to pixel is determined and the relative changes from pattern to pattern are plotted in this false colour map. Rotations of ca. $1^{\circ}$ are easily determined. If the local crystal is also strained (in addition to a rotation) this will be seen through geometric distortions to the patterns. That again may be measured and using a deformation matrix formalism the 'projected' strain components may be recovered. Figure 1(b) shows a shear strain map derived from a 4D SED data set. The strain is plotted on a false colour scale indicating tensile and compressive strain as a percentage [4]. For a fully 3-dimensional set of strain components a strain 
tomography approach is needed in which, in general, three independent tilt series of 4D maps are required - a formidable undertaking, but some early progress has been made [5]. Analyses of such data is significantly easier if each diffraction pattern is sensitive to only the in-plane strain / rotation. By applying precession, the Ewald sphere is rocked through the Bragg condition of any reflection in the pattern, integrating through the 'rocking curve', and, in doing so, making the final recorded pattern insensitive to out-of-plane tilts [4]. In addition, there is the added benefit that by rocking the Ewald sphere, higher order reflections may be intercepted and thus the precision with which the pattern is analysed may be improved [6].

\section{References:}

[1] H. Yang et al, Nature Commun. 7 (2016), p. 12532

[2] J.-L. Rouviere et al, Appl. Phys. Lett. 103 (2013), p. 24

[3] S. Kang, et al, Proc. European Microscopy Congress (2016), p. 692-693.

[4] T. Ostacevicius (2017) PhD Thesis, University of Cambridge

[5] D. Johnstone, A. van Helvoort and P.|A. Midgley, Microsc. Microanal. 23 (S1) (2017), p. 1710.

[6] The authors thank many people for useful discussions and contributions including S. Kang, H.

Jinnai, T. Ostasevicius, A. Ferrari, A. van Helvoort, J .Sunde, J. Barnard and F. de la Pena. We thank the EPSRC for funding: grant number EP/R008779.
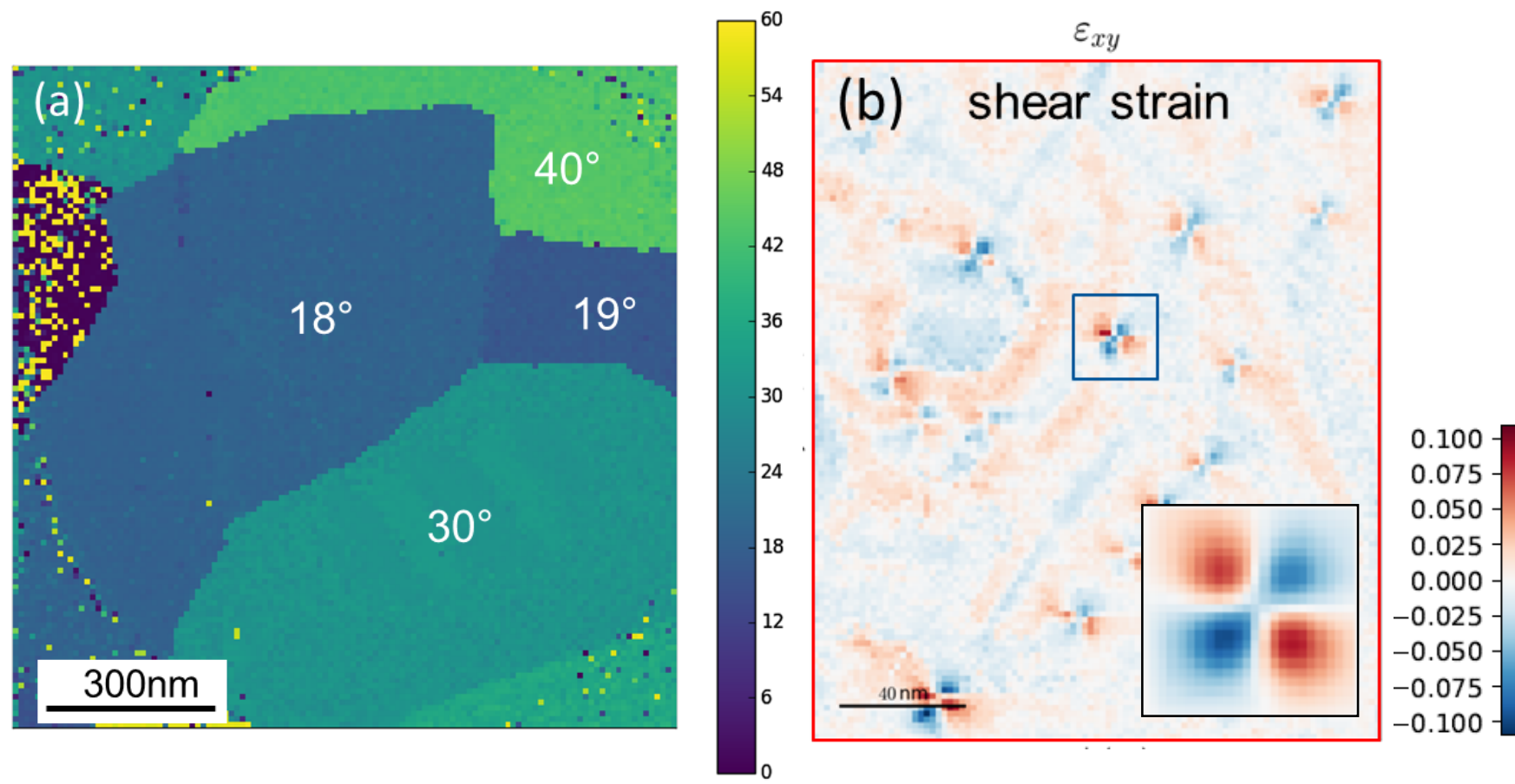

Figure 1. (a) Orientation map from CVD grown graphene derived from a 4D SED data set. Angles indicate relative orientations. (b) Part of a shear strain map from an Al 6xxx alloy showing distinctive strain profiles near to Al-Mg-Si precipitates. The inset shows an average strain map derived from tens of regions of near-identical precipitate orientation. The strain scale is a percentage [5]. 